Volume 8, No.1.3, 2019

International Journal of Advanced Trends in Computer Science and Engineering

Available Online at http://www.warse.org/IJATCSE/static/pdf/file/ijatcse0581.32019.pdf

https://doi.org/10.30534/ijatcse/2019/0581.32019

\title{
Energy-Efficient Routing Protocol for Node Lifetime Enhancement in Wireless Sensor Networks
}

\author{
Amairullah Khan Lodhi ${ }^{1}$ ， M S S Rukhmini ${ }^{2}$, Mohammad Iliyas ${ }^{3}$ ，Farha Anjum ${ }^{4}$ \\ ${ }^{1,2}$ D. Scholar, ECE Department, Vignan's Foundation for Science, Technology, \& Research \\ (Deemed to be university), Vadlamudi, Guntur, India \\ ${ }^{3}$ Professor \& Head, ECE Dept. Shadan College of Engineering \& Technology, Hyderabad, India \\ ${ }^{4}$ Professor, ECE Dept. Siddhartha Institute of Engineering \& Technology, Ibrahimpatnam, Hyderabad.
}

\begin{abstract}
Wireless sensor networks (WSNs) countenance some deceitful attack which may damage instructions and capacity in an elegant way and with irregular performance to fabricate the premier damage without being exposed. WSNs often consist of tiny devices with limited energy, computational power, transmission range, and memory. Energy is one of the most important resources in such networks. Therefore, optimal use of energy is necessary. In this paper, we present a novel energy-efficient routing protocol for WSNs. The suggested protocol may be hierarchic and group built. Every bunch comprises from claiming one cluster head $(\mathrm{CH})$ node, two agent $\mathrm{CH}$ nodes, also a percentage conventional sensor hubs. Those reclustering the long haul Also vitality necessities need been minimized Eventually Tom's perusing presenting the idea from $\mathrm{CH}$ board. Recent approaches use selective encryption to minimize energy consumption. WSNs are resource constrained. Moreover, exchange ways need aid utilized to information transmission the middle of An $\mathrm{CH}$ hub and the bs. Thorough reproduction effects portray those vitality efficiency, throughput, and prolonged lifetime of the hubs under that impact of the suggested protocol. Future scope from claiming this worth of effort will be delineated.
\end{abstract}

Key words: Wireless Sensor Network, mobile base station, Path tracing, mo-bile nodes, Energy efficiency, reliability, routing protocol, sensor nodes, Heterogeneous WSN.

\section{INTRODUCTION}

A Wireless Sensor Network (WSN) consists of the selfdirected nodes that are spread spatially for preservation of environmental or substantial states such as humidity, gravity, temperature, etc. WSN integrates an access, between the nodes and a client, to provide wireless connectivity to both the wireless distributed nodes and wired surroundings.

As shown the figure 1, the Common applications of WSNs are Health Care monitoring; Earth sensing, Forest fire detection, data recording, enemy intrusion detection and geo-fencing [1]. In this work, we provide a comprehensive survey on energy-efficient WSN protocols [2].

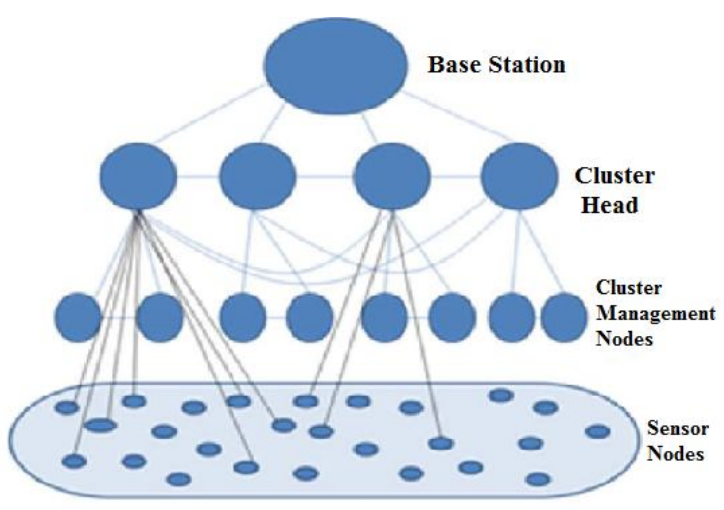

Figure 1: WSN System Basic Architecture

WSN schemes used in routing messages are classified into four categories i.e. Communication model, Network structure, Reliable routing schemes and topology-based routing schemes. It takes the amount of power and energy into consideration to minimize the energy consumption and to increase the lifetime of WSNs. We also discuss and compare their metrics such as scalability, mobility, and power usage [3].

\section{RELATED WORK}

The most important factor when developing WSN routing protocols is energy efficiency of a node, which has a direct impact on the network lifetime. Several surveys are there including current efforts and future work to develop energy efficient routing protocols. Some of those literatures on routing protocols are presented below with the discussion of comparison of existing protocols and our work.

A survey done on routing protocols on WSNs is discussed in [3] which classifies the routing protocols into three categories according to structure of the network: Flat, location-based and hierarchical infrastructure. These protocols are further classified into query-based, multipathbased, QoS (Quality of service) based and negotiation-based routing techniques according to protocol operations. Thus, the survey describes the limited supply of energy, computing power and bandwidth of the wireless sensor nodes along with the advantages and disadvantages of each routing protocol. In this work, we compare energy efficient routing protocols comprehensively focusing on energy efficiency issues to help the researchers on their work. 
In 2015, a few routing protocols were discussed in a survey [4] for WSNs and classified into hierarchical, data-centric and location-based protocols. The two considered primary energy metrics are:

Although, a good number of surveys for WSNs, WSN routing and WSN MAC algorithms is provided in reference. Our work gives an analytic survey over the energy-efficient WSN routing protocols. Our survey focuses on energyefficient WSN routing protocol to aid the readers in choosing the most appropriate energy-efficient routing protocol for Wireless sensor networks. We also discussed the strength and weakness of each protocol and compared each of them with some metrics such as Mobility, Scalability, Power usage, Route metric, robustness, QoS Support and periodic message type.

\section{PROPOSED WORK}

Initially, the requirements in defense applications drove the research and development of WSN routing protocols resulting in the development of many systems like Remote Battlefield Sensor System (REMBASS) and Low-flying aircraft acoustic tracking. A wireless sensor network that provides surveillance services in battlefield is provided in [5]. An sensor and more actuator system will be examined to advanced mobile homes will support elderly Also debilitated people Previously, [6]. When a disappointment may be distinguished in the neighborhood, those one-hop neighbors of the neglected performing artist might focus the impact, i. E. , if those fizzled hub may be basic on system connectivity. The CPRA serves that briefest way from claiming the greater part hubs.

Procedure Node Failure Detection (A)

1) If pulse message $[\mathrm{A}]<\min$

count c 3) \{

4) Failure node list $\leftarrow \mathrm{A}$; //Intimates dynamic sensor

5) Replace

node[A];6) \}

7) Else

8) Working node list $\leftarrow A$;

Fig 2. Check point recovery algorithm

\subsection{Selection of node for replacement}

A node is selected for replacement only if that node is nearest to the failure node. The node which is selected for replacement should have high energy and should be nearest to the failure node. The dynamic node replaces this node with the failure node. The energy loss of each node is detected by the static node.

\subsection{Node Replacement}

The node replacement is done by Network Topology Management (NTM). NTM helps maintain the link between the nodes. It maintains the link between the nodes when energy loss is detected in anode. During replacement there are possibilities for direct links between nodes to break NTM helps maintain the link. It maintains the link between the nodes without affecting the packet transmission. This process takes place continuously.

\subsection{Procedure Replace Node}

1) for each $n \in$ neighbour_list[A]

2) find link_count;

3) $\mathrm{T}=\min ($ link_count $)$;
4) if energy $[\mathrm{t}]>$ avg energy

5) $\mathrm{A}=\mathrm{T}$;

6) else

7) remove $\mathrm{T}$ from neighbour_list[A];

8) Replace node[A];

Fig 3. Procedure for node replacement

\section{SIMULATION RESULTS}

All simulations have been carried out using the NS simulator program me version 2.34. It is primarily UNIX based and uses TCL as its scripting language. NS-2 is a standard experiment environment in research community. However, wireless sensor networks are deployed mostly in open and unguarded environment. There are two types of WSN first, homogeneous WSN and second, heterogeneous WSN. We have chosen heterogeneous WSN for our survey because there are following advantages of heterogeneous WSN:

1. Prolonging network lifetime

2. Improving reliability of data transmission.

3. Decreasing latency of data transportation. These qualities are not present in homogeneous WSN.

\subsection{DSDV Route Discovery}

DSDV protocol will be In light of those Bellman-Ford directing calculation. It is a proactive protocol furthermore belongs of the table- determined gang. Routes between those hubs in the organize would continuously being supported Furthermore updated. Every hub in the system administers An directing table which holds data something like how of age those course is, those most brief separation and in addition those initial hub on the briefest way to each other hub in the WSNs. Late methodologies utilize AODV for WSN, but even though the overhead is high in DSDV because of being a proactive protocol the energy consumption is less when compared with protocols like AODV, DSR, and AOMDV [7].

\subsection{Failure node detection}

Node failure is caused by two reasons - energy loss in the node up to a point where it cannot transmit anymore or the node has been captured by the attacker and behaves as a malicious node. A node that fall in any of these categories is labeled as failure nodes. Two methods are used to detect node failure.

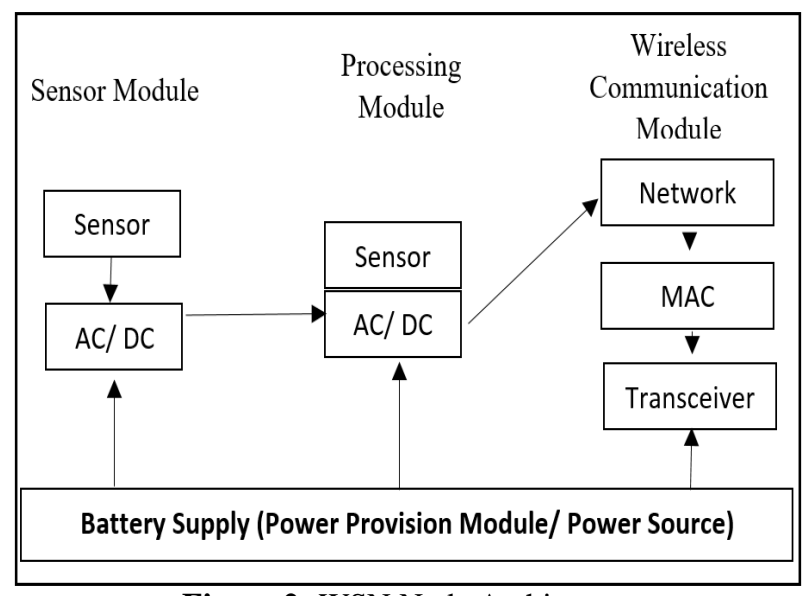

Figure 2: WSN Node Architecture 
As shown in the Figure 2, several modules are present in WSN nodes: Power Supply Module, Sensor Module, Wireless Communication Module and Processing Module. In [8] the authors presented a realistic model of power consumption for WSN by involving the characteristics of a low power transceiver.

Another model with more updated approaches regarding WSN node energy consumption based on as shown in figure 3 , depends upon its components and it is summarized below:

\subsection{Sensor Module}

In this module, the energy consumption is also related to the sense operation (Sleep/wake, Periodic, etc.) of the node. In particular, the consumption of energy in periodic mode is modeled as:

The $e_{o n / o f f}$ in this relation is the one-time energy consumption of operation of a closing sensor, $\mathrm{e}_{\text {off } / \mathrm{on}}$ is Those one-time Vitality utilization for operation about an opening sensor What's more e sensor/run is those sensing operation Vitality utilization which will be equivalent to those working voltage increased with the sensor current Furthermore interim from claiming sensing operation.

\subsection{Processing Module}

Sensor controlling, data processing and the protocol communication are the primary activities of this module. The module supports three operation states in most cases i.e. idle, sleep and run. The energy consumption of a processor is denoted as $e_{\text {cpu. }}$ Where $e_{\text {cpu }}$ is the sum of state energy consumption $\mathrm{e}_{\mathrm{cpu} / \text { state }}$ and energy consumption in state transition $\mathrm{e}_{\mathrm{cpu} / \text { change. }}$ Consider $\mathrm{a}=1,2,3,4 \ldots \mathrm{x}$ to is the operation state of a processor ( $\mathrm{m}$ is no. of processor state) and $b=1,2,3,4 \ldots y$ is the type of state (where $n$ is the no. of state transition).

Then

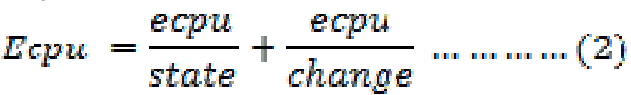

$$
\begin{aligned}
& \text { Eopu }= \\
& \frac{\frac{\Delta=2}{\operatorname{sen} s}(a)-\sec }{\sec }(a)+
\end{aligned}
$$

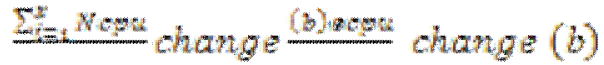

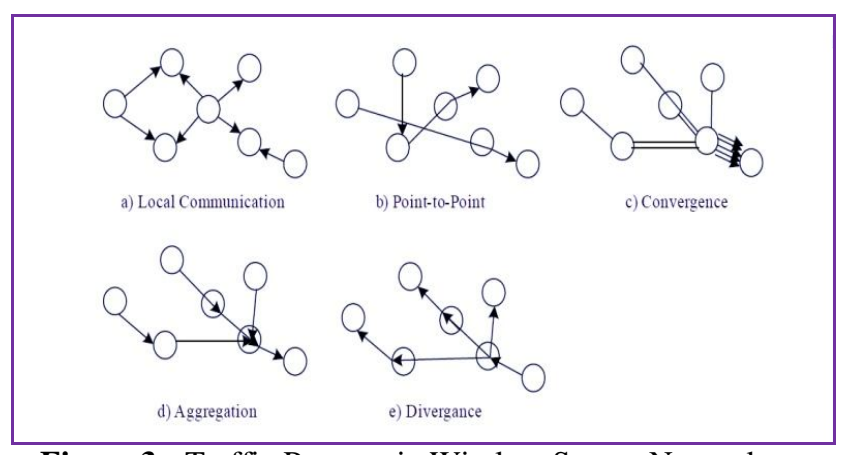

Figure 3: Traffic Patterns in Wireless Sensor Networks

In this relation, $\mathrm{P}_{\text {cpu/state }}(\mathrm{a})$ is the power of state $a$, which can be found from reference manual. The time interval $T_{\text {cpu/state }}$ (a) of the state $a$ is a statistical variable. The frequency of transition of state $b$ is denoted by $\mathrm{N}_{\text {cpu/change }}(\mathrm{b})$ and $\mathrm{e}_{\mathrm{cpu} / \text { change }}$ (b) denote the consumption of energy in one-time state transition $b$.

\subsection{Power supply module}

For each node, the power supply module is related to its model and manufacturer. On receive mode, the current draw is $16 \mathrm{~mA}$ and consumption on transmit is $10 \mathrm{~mA}, 13 \mathrm{~mA}$ and $17 \mathrm{~mA}$ respectively for $\mathrm{T}_{\mathrm{x}}$ value of $-17 \mathrm{dBm},-3 \mathrm{dBm}$ and $+3 \mathrm{dBm}$. Another node known as MICA2 is also equipped with AA batteries and consumes $27 \mathrm{~mA}$ with maximum power on transmit and receives $10 \mathrm{~mA}$ at an average. Also, it consumes less than $0.001 \mathrm{~mA}$ in sleep mode.

\subsection{Classification of WSN routing techniques}

The following characteristics make WSN routing more demanding when compared to other wireless networks such as WSNS or other cellular networks:

Here are severe constraints in processing, storage and energy capacities resulting in demand of careful resource management of sensor nodes

He flow of data packets from multiple nodes to a base station is required by almost all applications

SN nodes are stationary mostly after their deployment resulting in non-frequent and predictable topological adjustments

He collected data might present unenviable redundancy which needs to be exploited by the routing algorithms to improve utilization of energy and bandwidth ata collection is based upon location under normal states, which makes position awareness of sensors important. It is possible to use GPS for this purpose but its independent solutions are favorable for WSN [10] location problems.

\subsection{Current processing condition (CPC) of a node}

To discover an energy efficient route from source to destination, we proposed a new algorithm known as "Energy-aware [11] Proactive routing for WSNs based on Knapsack algorithm." This system selects a route according to the current processing condition of a node to achieve the link reliability and the amount of stored energy in terms of energy and input buffer. Our work proposed a major handout which is classified below:

Minimum energy needed to process packets in kilobytes Current processing condition of a node in terms of energy and input buffer

Node priority according to value of threshold route selection based on node priority

A node satisfies the threshold condition to participate in routing in terms of energy. To prevent a node from becoming a bottleneck, the optimized information capacity of a metric node is used with respect to traffic and residual energy.

To calculate the current processing condition (CPC) of a node with respect to traffic and residual energy, consider a node $\mathrm{N}_{\mathrm{x}}$ with buffer $\mathrm{B}$ (Kilobytes)and energy $\mathrm{e}_{\mathrm{x}}$ (Joules) processing packets of ' $\mathrm{x}$ ' kilobytes.

The equation given below shows the energy e $\left(K_{n}\right)$ within a multi hop wireless sensor network required by a node. $e\left(k_{x}\right)=t\left(k_{x}\right)+r\left(k_{x}\right)+d\left(k_{x}\right)$

Where, $\quad t\left(k_{s}\right) \| r$ send, receive and process energy of a node in $\mathrm{k}_{\mathrm{x}} \mathrm{kbps}$. The packets are processed through a node $\mathrm{N}_{\mathrm{x}} \mathrm{kb}$, where $\mathrm{x}=1,2,3 \ldots$ The partial packet processing is impossible and hence the 
packets must be completely processed. To achieve optimum results, we designed two dimensional array using knapsack algorithms. Algorithm for calculation of current processing condition of a node is given below:

$$
\begin{aligned}
& S[0 \ldots n, 0 \ldots e x] \\
& \quad \forall 0 \leq e(k x) \leq e ; 1 \leq x \leq n
\end{aligned}
$$

Where, equation $S S[t(k x), c x]$ with " $k x$ "

Kilobits of data process maximum amount of packets (Where $\mathrm{x}=1,2,3, \ldots$ )

The given equation calculates current processing condition of a node

$S[0, e(k x)]=0 ; 0 \leq e(k x) \leq e x$ no packet process through the node

Optimization is given below: $s[i, e(k x)]=\max (s[x-1, e(k x)] s x+s[x-1 \cdot e(k)$ $\forall 1 \leq I \leq x$ and $0 \leq e(k x) \leq e x$

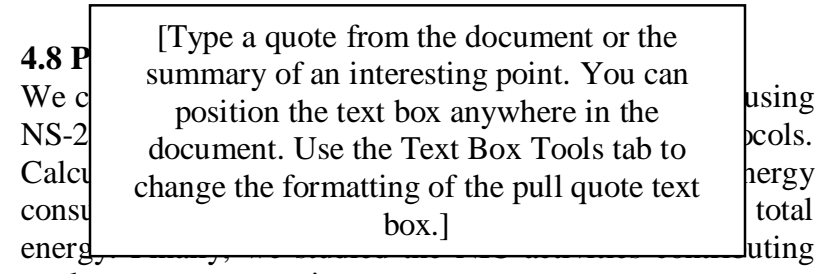
total energy consumption.

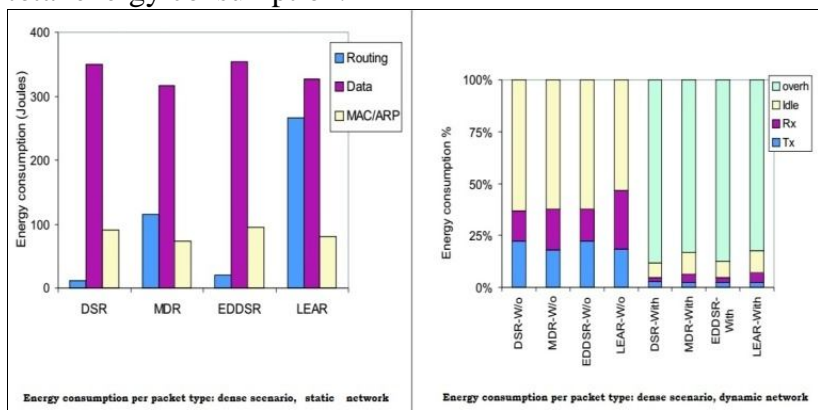

Figure 4: Comparison of expiry of number of nodes over time after battery capacity expire

As in figure 4 above provides the comparison of expiry of number of nodes over time after the battery capacity expire in a static and dynamic environment. A similar behavior is shown in all protocols but EDDSR shows a slight improvement at the end of simulation. In a scenario of dynamic network, MDR and EDDSR schemes are better than DSR and LEAR mechanism, which consume energy in large amount and induce more energy consumption. This effect is due to frequent route recovery process that uses DDREQ packets.

\section{CONCLUSION}

WSNs are emerging these days and playing a vital role in efficient selection and delivery of data. Energy efficiency is an important challenge in WSNs, which are equipped with batteries having limited storage capacities. Thus, energy efficient routing techniques are required in corporate operations of WSNs to provide the network connectivity and routing of data with less energy required.

In this work, we focused on energy efficient protocols developed for Wireless sensor networks and classified them to obtain an ideal solution. The flat protocols are ideal for fixed nodes and small network infrastructure and may not work feasibly due to overhead. On the other hand, hierarchical protocols are suitable for sensor networks with high coverage area and heavy load. The negotiation-based protocols can perform optimally in broadcast and point-topoint networks. The emergence of next generation Wireless sensor networks and personal communication systems has led to occurrence of new type of scheme based on mobile agent. Routing protocols based on mobile agent have mobile agent, software or program migrating among the network nodes to perform task intelligently and anonymously according to environmental conditions.

\section{REFERENCES}

1. Devi, Gayatri, Rajeeb Sankar Bal, and Sasmita Manjari ) Nayak. "Node Deployment and Coverage in Wireless Sensor Network." International Journal of Innovative Research in Advanced Engineering (IJIRAE) 2, no. 1 (2015): 139-145

https://doi.org/10.1007/978-3-662-57277-1_10

2. Bhushan, Bharat, and G. Sahoo. "Routing Protocols in Wireless Sensor Networks." In Computational Intelligence in Sensor Networks, pp. 215-248. Springer, Berlin, Heidelberg, 2019.

3. Lodhi, Amairullah Khan, and Syed Abdul Sattar. "Cluster Head Selection by Optimized Ability to Restrict Packet Drop in Wireless Sensor Networks." In Soft Computing in Data Analytics, pp. 453-461. Springer, Singapore, 2019.

https://doi.org/10.1007/978-981-13-0514-6_45

4. Lodhi, Amairullah Khan, and Syed Abdul Sattar. "Cluster Head Selection by Optimized Ability to Restrict Packet Drop in Wireless Sensor Networks." In Soft Computing in Data Analytics, pp. 453-461. Springer, Singapore, 2019. https://doi.org/10.1007/978-981-13-0514-6_45

5. Lin, Kai, Fuzhen Xia, Chensi Li, Di Wang, and Iztok Humar. "Emotion-aware system design for the battlefield environment." Information Fusion 47 (2019): $102-110$

https://doi.org/10.1016/j.inffus.2018.07.008

6. Mayer, Joceli. "Low Cost Automatic Speech Recognition IOT Architecture for Hands Free Assistance to People with Mobility Restrictions." In Proceedings on the International Conference on Internet Computing (ICOMP), pp. 53-58. The Steering Committee of The World Congress in Computer Science, Computer Engineering and Applied Computing (World Comp), 2018.

7. Tarique, Mohammed, and Rumana Islam. "Performances of Ad Hoc Networks Under Deterministic and Probabilistic Channel Conditions: Cases for Single Path and Multipath Routing Protocols." International Journal of Computer Networks and Communications (IJCNC) 10, no. 4 (2018): 1-21. https://doi.org/10.5121/ijenc.2018.10401

8. Brini, Oussama, Dominic Deslandes, and Frederic Nabki. "A System-Level Methodology for the Design of Reliable Low-Power Wireless Sensor Networks." Sensors 19, no. 8 (2019): 1800. https://doi.org/10.3390/s19081800 
9. Shi, Hongling, Kun Mean Hou, Xunxing Diao, Liu Xing, Jian-Jin Li, and Christophe De Vaulx. "A wireless multimedia sensor network platform for environmental event detection dedicated to precision agriculture." arXiv preprint arXiv: 1806.03237 (2018).

10. Nouf Saeed Alotaibi," An Efficient Technique for Reducing Transmission Energy of Sensor Node to Enhance the Lifetime of the WSN's", International Journal of Advanced Trends in Computer Science and Engineering, Volume 8, No.2, March - April 2019. https://doi.org/10.30534/ijatcse/2019/02822019

11. B. Chandirika1, N. K. Sakthivel2 S. Subasree," An Energy Efficient K-Means Clustering based Trust
Model for Wireless Sensor Networks", International Journal of Advanced Trends in Computer Science and Engineering, Volume 8, No.2, March - April 2019. https://doi.org/10.30534/ijatcse/2019/08822019

12. Papa Rao Nalajala, Rotala Umarani, Naroju Mounika, "Design Of Intelligent Road Traffic Control System For Ambulance Using RF And GSM Technology ", International Journal of Advanced Trends in Computer Science and Engineering, Vol. 5, No. 1, 2016. 